\title{
AIN MEMS filters with extremely high bandwidth widening capability
}

\author{
Anming Gao', Kangfu Liü, Junrui Liang ${ }^{2,3}$ and Tao Wu (id)
}

\begin{abstract}
This paper presents radio frequency (RF) microelectromechanical system (MEMS) filters with extremely high bandwidth widening capability. The proposed filtering topologies include hybrid configurations consisting of piezoelectric MEMS resonators and surface-mounted lumped elements. The MEMS resonators set the center frequency and provide electromechanical coupling to construct the filters, while the lumped-element-based matching networks help widen the bandwidth (BW) and enhance the out-of-band rejection. Aluminum nitride (AIN) SO Lamb wave resonators are then applied to the proposed filtering topologies. AIN SO first- and second-order wideband filters are studied and have shown prominent performance. Finally, the AIN SO first-order wideband filter is experimentally implemented and characterized. The demonstrated first-order filter shows a large fractional bandwidth (FBW) of 5.6\% (achieved with a resonator coupling of 0.94\%) and a low insertion loss (IL) of $1.84 \mathrm{~dB}$. The extracted bandwidth widening factor (BWF) is 6, which is approximately 12 times higher than those of the current ladder or lattice filtering topologies. This impressive bandwidth widening capability holds great potential for satisfying the stringent BW requirements of bands $n 77, n 78$, and $n 79$ of $5 \mathrm{G}$ new radio (NR) and will overcome an outstanding technology hurdle in placing $5 \mathrm{G} \mathrm{NR}$ into the marketplace.
\end{abstract}

\section{Introduction}

The proliferation of $5 \mathrm{G}$ communications has led to the allocation of wider bands for faster data transmission and processing ${ }^{1}$. Radio frequency (RF) bandpass filters, which are used to select the desired signals while suppressing the unwanted signals, are the keys to define these frequency bands. Therefore, the realization of filters with a wide bandwidth (BW) is attracting tremendous attention. Filters based on various technologies, including microstrip, metal cavity, dielectric, lumped element, and acoustic or microelectromechanical systems (MEMS), have all been developed in recent years. Among these technologies, MEMS filters stand out for their compact size, low cost, and excellent filtering performance (especially the sharp

Correspondence: Tao Wu (wutao@shanghaitech.edu.cn)

${ }^{1}$ Department of Electrical and Computer Engineering, University of Illinois at Urbana-Champaign, Urbana, IL 61820, USA

${ }^{2}$ School of Information Science and Technology (SIST), ShanghaiTech University, Shanghai 201210, China

Full list of author information is available at the end of the article roll-off). These advantages make MEMS filters the most promising solution to 5G filtering applications. Researchers have made extensive investigations of MEMS filtering technologies in three main directions: exploiting resonant modes with higher coupling $\left(k_{\mathrm{t}}^{2}\right)^{2-4}$, exploring strong piezoelectricity materials (e.g., $\mathrm{AlScN}^{5-7}$, $\mathrm{LiNbO}_{3}{ }^{1}$ ), and hybridizing acoustic resonators to expand the bandwidth ${ }^{8-10}$. For example, the shear horizontal (SH0) mode and first-order asymmetric (A1) mode in lithium niobate $\left(\mathrm{LiNbO}_{3}\right)$ have been demonstrated with high $k_{\mathrm{t}}^{2}$ values of $50 \%$ and $22 \%$, respectively ${ }^{11-13}$. Acoustic resonators with these modes can be used to construct wideband filters ${ }^{14,15}$. However, the severe power-handling issue and temperature instability make the two modes still not promising enough for wideband or high-frequency applications. Nevertheless, AIN is the first choice in piezoelectric resonators ${ }^{16-18}$ and sensors ${ }^{19}$ since it has a high power-handling capability and excellent thermal stability and offers a mature process that can be integrated with complementary metal-oxide-semiconductor (CMOS) 
integrated circuits $(\mathrm{ICs})^{20}$. Scandium (Sc) has been studied for its doping with AlN to increase the piezoelectric coefficients that determine the couplings of the resonators ${ }^{21,22}$. It has been reported that doped $\mathrm{Sc}_{0.12} \mathrm{Al}_{0.88} \mathrm{~N}$ can increase the piezoelectric coefficient $d_{33}$ by $50 \%$ and reduce the stiffness constant $c_{33}$ by $10 \%{ }^{23}$. Thanks to this achievement, $k_{\mathrm{t}}^{2}$ is improved by 1.7 times. Nevertheless, the doped AIN always comes with the degradation of the quality factor, which directly affects the insertion loss (IL) of the filters. In addition to the two developments above, researchers have also made efforts to hybridize filters using quartz surface acoustic wave (SAW) resonators and lumped elements or microwave transmission lines ${ }^{8-10}$. However, these hybridized filters suffer from small bandwidth (BW), poor rolloff, and low out-of-band rejection.

To solve the above issues, this paper proposes AlN MEMS filters with extremely high bandwidth widening capabilities. The wideband filters utilize AlN Lamb wave resonators in conjunction with surface-mounted lumped elements. Compared with conventional ladder and lattice topologies, the proposed filters show a much larger bandwidth widening capability.

This paper is organized as follows: the Method and Modeling section presents the models of the two filtering topologies: first-order and second-order wideband filters. Both filters are designed based on a general MEMS resonator. The Material and Device section illustrates the AIN MEMS first-order and second-order wideband filters. It begins with the introduction of the AlN S0 Lamb wave resonator, followed by the implementation of the two wideband filtering topologies. The Results and Discussion section shows the experimental realization of the AlN first-order wideband filter. The filter fabrication, assembly, and characterization results are covered. The final section concludes this paper with some potential future endeavors.

\section{Method and modeling}

\section{First-order wideband filter}

The filter construction starts with the MEMS resonator. The electrical response of a MEMS resonator is often represented with the modified Butterworth-Van Dyke (MBVD) model, in which $C_{0}$ is the static capacitance of the resonator and $L_{\mathrm{m}}, C_{\mathrm{m}}$, and $R_{\mathrm{m}}$ are the motional inductance, motional capacitance, and motional resistance, respectively. Extraction of the MBVD parameters can be found in many literature reports ${ }^{24-29}$. The motional branch forms the mechanical resonance equivalently in the electrical domain via piezoelectricity. As an example, the measured response of a MEMS resonator is plotted and fitted in Fig. 1a. The demonstrated resonator has a typical resonant frequency $f_{\mathrm{s}}$ and an antiresonant frequency $f_{\mathrm{p}}$. The quality factor and the piezoelectric coupling factor are two important parameters for the performance of the resonators, which can be related to the MBVD model parameters. Therefore, the MBVD model is extracted from the measured response and will be used as the basis for the following filter analysis.

As shown in Fig. 1d, the proposed first-order filter is obtained from the combination of the topology in Fig. 1b, c, which consists of a MEMS resonator, a parallel inductor $L_{0}$, and a pair of three-lumped-element matching networks, which includes a series inductor $L_{\mathrm{s}}$, a shunt inductor $L_{\mathrm{p}}$, and a shunt capacitor $C_{\mathrm{p}}$. When the MEMS resonator parallel with the inductor $L_{0}$, two symmetric transmission zeros $f_{\mathrm{a} 1}$ and $f_{\mathrm{a} 2}$ are generated, as indicated in Fig. 1b. The filter is centered at the same resonant frequency $\left(f_{\mathrm{s}}\right)$ as the MEMS resonator, and it features a large bandwidth and two deep transmission zeros (TZs).

The series resonance frequency $f_{\mathrm{s}}$ is the frequency where $L_{\mathrm{m}}$ and $C_{\mathrm{m}}$ cancel each other out, while the antiresonance frequency $f_{\mathrm{p}}$ arises when $C_{0}$ and $C_{\mathrm{m}}$ collectively cancel out $L_{\mathrm{m}}$. The parallel $L_{0}$ is added to decouple the antiresonance and generate two symmetric TZs: $f_{\mathrm{a} 1}$ and $f_{\mathrm{a} 2}$. The two TZs are determined by the MBVD model and $L_{0}$, as shown in Fig. $1 \mathrm{~b}$ and computed as

$$
f_{\mathrm{a} 1, \mathrm{a} 2}=\frac{1}{2 \pi} \sqrt{\frac{\omega_{\mathrm{s}}^{2} \mp \sqrt{t_{1} t_{2}}+\omega_{\mathrm{s} 0}^{2}+C_{\mathrm{m}} L_{0} \omega_{\mathrm{s}}^{2} \omega_{\mathrm{s} 0}^{2}}{2}}
$$

where

$$
\begin{aligned}
& t_{1}=C_{\mathrm{m}} L_{0} \omega_{\mathrm{s}}^{2} \omega_{\mathrm{s} 0}^{2}+\omega_{\mathrm{s}}^{2}-2 \omega_{\mathrm{s}} \omega_{\mathrm{s} 0}+\omega_{\mathrm{s}}^{2} \\
& t_{2}=C_{\mathrm{m}} L_{0} \omega_{\mathrm{s}}^{2} \omega_{\mathrm{s} 0}^{2}+\omega_{\mathrm{s}}^{2}+2 \omega_{\mathrm{s}} \omega_{\mathrm{s} 0}+\omega_{\mathrm{s}}^{2} \\
& \omega_{\mathrm{s}}=1 / \sqrt{C_{\mathrm{m}} L_{\mathrm{m}}} \\
& \omega_{\mathrm{s} 0}=1 / \sqrt{C_{0} L_{0}}
\end{aligned}
$$

If we set, $\omega_{\mathrm{s}}=\omega_{s 0}$, then

$$
L_{0}=\frac{L_{\mathrm{m}} C_{\mathrm{m}}}{C_{0}}=\frac{1}{\omega_{\mathrm{s}}^{2} C_{0}}=\frac{1}{\left(2 \pi f_{\mathrm{s}}\right)^{2} C_{0}}
$$

Then, Eq. (1) can be rewritten as

$$
f_{\mathrm{a} 1, \mathrm{a} 2}=\frac{\omega_{\mathrm{s}}}{2 \pi} \sqrt{\frac{2+C_{\mathrm{m}} / C_{0} \pm \sqrt{\left(2+C_{\mathrm{m}} / C_{0}\right)^{2}-4}}{2}}
$$

Since the piezoelectric coupling factor of the resonator device is proportional to $C_{\mathrm{m}} / C_{0}{ }^{3}$ and the TZs determine the maximum bandwidth of the filter, Eq. (3) implies that a higher bandwidth widening capability can be obtained by using resonators with a larger piezoelectric coupling factor. 

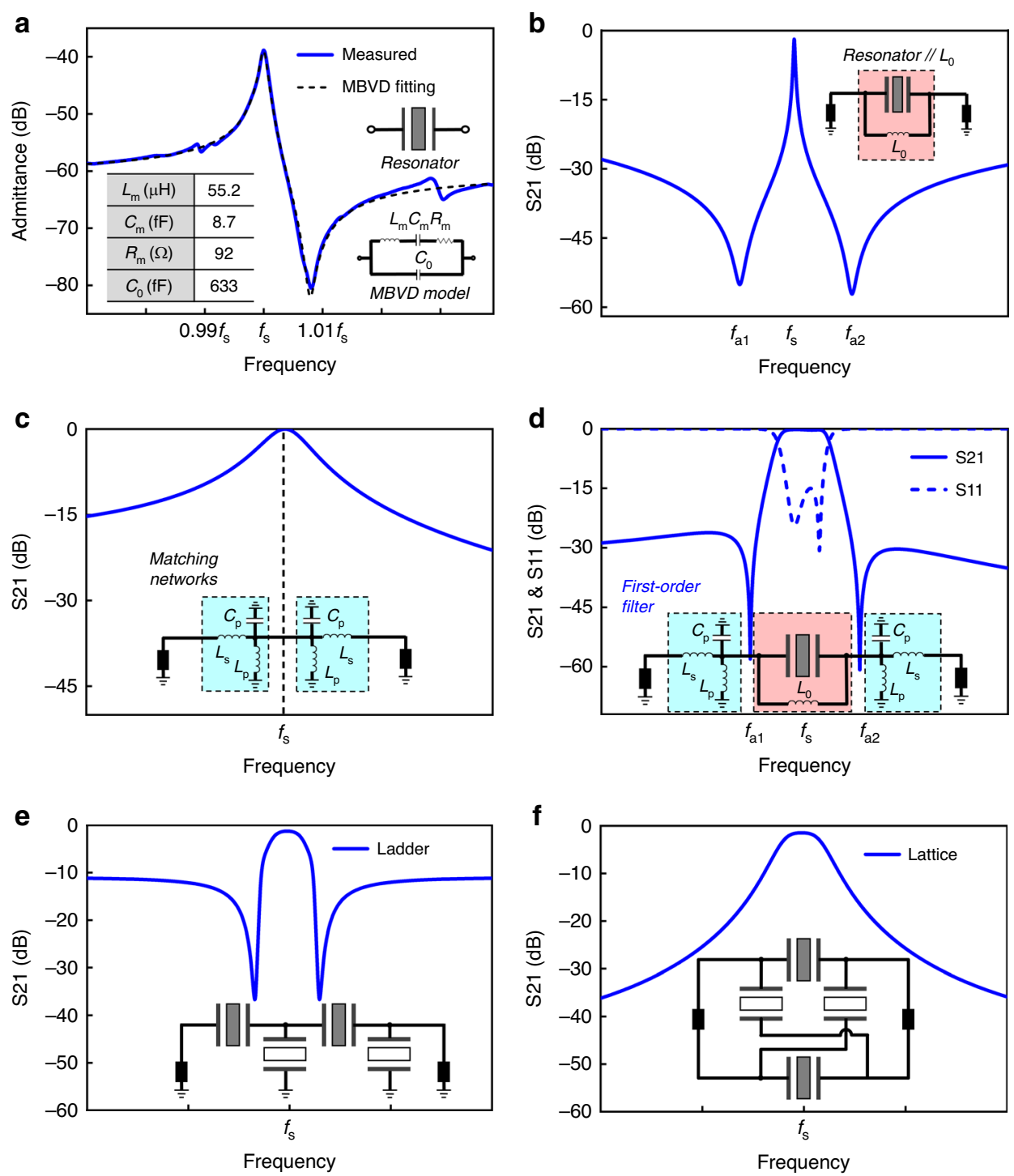

Fig. 1 The topology and principle of the proposed first-order filter. a MEMS resonator representation and its MBVD fitting model. The admittance response is fitted with MBVD parameters. $\mathbf{b}$ The MEMS resonator and the paralleled inductor $L_{0}$. Two symmetric transmission zeros are generated. The center frequency is the same as the resonant frequency $\left(f_{s}\right)$ of the MEMS resonator. $\mathbf{c}$ Two three-lumped-element matching networks. Each consists of a series inductor, a shunt inductor, and a shunt capacitor. The two matching networks behave like a wide bandpass filter centered at $f_{s}$. $\mathbf{d}$ Proposed first-order wideband filter. It is constructed from the combination of the topologies in (b) and (c). The filter is centered at $f_{s}$. It features a large bandwidth and two deep TZs. e The frequency response and topology of a ladder-type filter. $\mathbf{f}$ The frequency response and topology of a lattice-type filter

As demonstrated in Fig. 1c, the lumped-element matching network consists of a series inductor $L_{\mathrm{s}}$, a shunt inductor $L_{\mathrm{p}}$, and a shunt capacitor $C_{\mathrm{p}}$. The purpose of the matching networks is to widen the bandwidth of the filter. The three-lumped elements resonate at $f_{\mathrm{s}}$ and are regulated by

$$
\left(\frac{1}{L_{\mathrm{p}}}+\frac{1}{L_{\mathrm{s}}}\right) \cdot \frac{1}{C_{\mathrm{p}}}=\omega_{\mathrm{s}}^{2}
$$

Only when $L_{\mathrm{s}}, L_{\mathrm{p}}$, and $C_{\mathrm{p}}$ obey Eq. (4) can we obtain a functional matching network. If any two of three are determined, then the third one can be acquired by Eq. (4). Therefore, only two parameters are independent, and the left parameter is dependent. In later discussion, $L_{\mathrm{s}}$ and $L_{\mathrm{p}}$ will be chosen as independent variables and $C_{\mathrm{p}}$ as the dependent variable.

Finally, as shown in Fig. 1d, the proposed filter is formed by combining the two topologies in Fig. 1b, c. In this filtering topology, the MEMS resonator and $L_{0}$ provide 

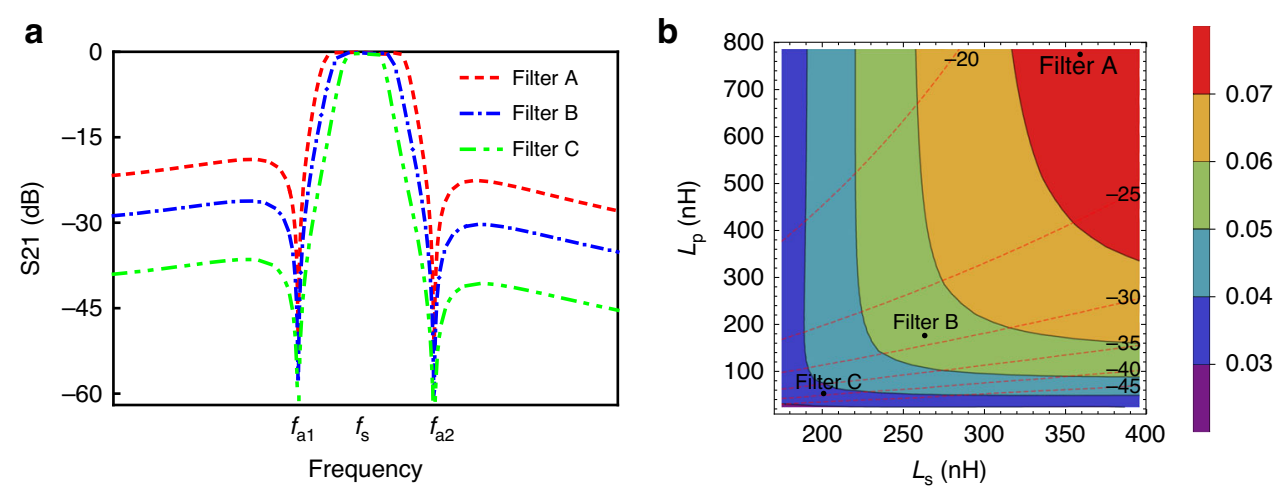

\begin{tabular}{c|c|c|c}
\hline C & & \\
\hline Parameters & Filter A & Filter B & Filter C \\
\hline$L_{0}(\mathrm{nH})$ & 750 & 750 & 750 \\
\hline$L_{\mathrm{s}}(\mathrm{nH})$ & 359 & 263 & 201 \\
$L_{\mathrm{p}}(\mathrm{nH})$ & 774 & 176 & 53 \\
$C_{\mathrm{p}}(\mathrm{pF})$ & 2.0 & 4.5 & 11.4 \\
\hline FBW & $7.2 \%$ & $5.4 \%$ & $3.7 \%$ \\
Rej $(\mathrm{dB})$ & 22 & 29 & 39 \\
\hline
\end{tabular}

\begin{tabular}{c|c|c|c}
\hline d Filter & $\begin{array}{c}\text { Resonator } \\
\text { coupling }\end{array}$ & FBW & BWF \\
\hline A, this work & $1.7 \%$ & $7.2 \%$ & 4.2 \\
B, this work & $1.7 \%$ & $5.4 \%$ & 3.2 \\
C, this work & $1.7 \%$ & $3.7 \%$ & 2.2 \\
Ladder & $1.7 \%$ & $0.78 \%$ & 0.45 \\
Lattice & $1.7 \%$ & $0.95 \%$ & 0.56 \\
\hline
\end{tabular}

Fig. 2 Simulated performance proposed first-order filter. a Simulated filtering performance of three first-order filters with different matching networks or matching lumped elements. Filter $A$ has the largest $L_{s}$ and $L_{p}$, Filter $B$ has an intermediate $L_{s}$ and $L_{p}$ and Filter $C$ has the smallest $L_{s}$ and $L_{p}$. b Simulated filtering FBW performance versus different $L_{s}$ and $L_{p}$; the colored solid contour lines are the boundaries for the FBW performance of the filter topology, while the dashed lines are boundaries for out-of-band rejection performance. $\mathbf{c}$ Parameters and comparison of the three first-order filters. $\mathbf{d}$ Comparison of the bandwidth widening effect of three first-order filters and conventional ladder and lattice filtering topologies

sharp roll-off, TZs, and set the center frequency of the filter, while the matching network, which in essence is an LC bandpass filter, offers the wide bandwidth for the filter. The filtering topology enjoys high-performance flexibility by tuning the lumped elements in the matching networks. As shown in Fig. 2b, when $L_{\mathrm{s}}$ and $L_{\mathrm{p}}$ are given a different value while $C_{\mathrm{p}}$ is determined by Eq. (4) $L_{\mathrm{s}}$ and $L_{\mathrm{p}}$ have different effects on the filter bandwidth and out-of-band rejection. In general, larger $L_{\mathrm{s}}$ and $L_{\mathrm{p}}$ lead to a greater fractional bandwidth (FBW), while $L_{\mathrm{p}}$ has a greater impact on out-of-band rejection than $L_{\mathrm{s}}$, and a smaller $L_{\mathrm{p}}$ corresponds to greater out-of-band rejection. $Q_{s}$ of the resonator and the lumped elements together determine the IL of the filter. By leveraging $L_{\mathrm{s}}$ and $L_{\mathrm{p}}$, it is possible to achieve wide bandwidth and excellent out-of-band rejection simultaneously.

As an example of the tunability, Fig. 2a demonstrates three filters (named Filters A, B, and C) with different matching networks (for quantification purposes, we assume $f_{\mathrm{s}}=229 \mathrm{MHz}$ and $f_{\mathrm{p}}=231 \mathrm{MHz}$ based on a measured MEMS resonator; $k_{\mathrm{t}}^{2}$ is then computed to be 1.7\%). The values of the three-lumped elements $L_{\mathrm{s}}, L_{\mathrm{p}}$, and $C_{\mathrm{p}}$ are listed in Fig. 2c. Among the three filters, Filter A has the largest $L_{\mathrm{s}}$ and largest $L_{\mathrm{p}} . C_{\mathrm{p}}$ is therefore the smallest. As explained above, a larger $L_{\mathrm{s}}$ and $L_{\mathrm{p}}$ produce a wider $\mathrm{BW}$, and a larger $L_{\mathrm{p}}$ leads to smaller out-of-band rejection. Therefore, Filter A is assumed to have the largest BW and the smallest out-of-band rejection, which is verified by the simulation in Fig. 2a. Filter $C$ has the smallest $L_{\mathrm{s}}$ and $L_{\mathrm{p}}$, so it has the smallest BW and the largest out-of-band rejection. The comparison of the performance of the three filters is concluded in Fig. 2c. Figure $2 \mathrm{~b}$ illustrates the simulated filtering FBW performance versus different $L_{\mathrm{s}}$ and $L_{\mathrm{p}}$ values; the dashed lines show the out-of-band rejection values for guidance.

The proposed first-order filters stand out for their wide FBWs. To justify their bandwidth widening capabilities, the three filters (A, B, and $\mathrm{C}$ ) are compared with existing widely used ladder and lattice filtering topologies. The bandwidth widening factor (BWF), defined as the quotient of the FBW and the resonator's electromechanical coupling, is adopted for evaluation. Conventional ladder and lattice topologies ${ }^{27}$, which are shown in Fig. 1e, f, typically achieve FBWs of $1 / 3-1 / 2$ of the resonator's coupling ${ }^{27,28}$. Taking the assumed MEMS resonator that has an electromechanical coupling of $1.7 \%$, for example, the FBWs of the ladder and lattice topologies are $0.78 \%$ and $0.95 \%$, respectively. Figure $2 \mathrm{~d}$ compares the BWFs of the three first-order filters and the ladder and lattice topologies. Apparently, the proposed first-order filters have the largest BWF of 4.2, which is 9.1 and 7.5 times higher than the ladder and lattice topologies, respectively. 
Though showing a large FBW, the roll-off and out-ofband rejection of the first-order wideband filter still needs enhancement. For further improvement, a second-order wideband filter is then proposed.

\section{Second-order wideband filter}

As shown in Fig. 3a, the second-order wideband filter is composed of two series of first-order wideband filters. The two first-order wideband filters are designed to be image symmetrical for simultaneous matching of the two ports. In order to have more freedom of matching impedance, one side of the matching network is changed to be $L_{\mathrm{s} 2}, L_{\mathrm{p} 2}$, and $C_{\mathrm{p} 2}$. The roles of $L_{0}, L_{\mathrm{s} 1}, L_{\mathrm{p} 1}, C_{\mathrm{p} 1}, L_{\mathrm{s} 2}$, $L_{\mathrm{p} 2}$, and $C_{\mathrm{p} 2}$ are the same as those described in the firstorder wideband filter, and they are also regulated by Eqs. (2) and (4). The second-order wideband filter features a much better performance of out-of-band rejection and roll-off. In addition, the FBW has also been improved. The simulated response of the second-order wideband filter in Fig. 3b demonstrates a large FBW of $6.7 \%$ and an extremely high out-of-band rejection of over $60 \mathrm{~dB}$. Moreover, the roll-off is also significantly enhanced, and the $-30 \mathrm{~dB}$ shape factor is only 1.05 .

The second-order wideband filter enjoys favorable performance flexibility since it has four tuning elements $\left(L_{\mathrm{s} 1}, L_{\mathrm{p} 1}, L_{\mathrm{s} 2}\right.$, and $\left.L_{\mathrm{p} 2}\right)$, while the first-order filter has only two tuning elements $\left(L_{\mathrm{s}}\right.$ and $\left.L_{\mathrm{p}}\right)$. Similarly, to demonstrate the tuning capability, another three second-order wideband filters (Filters D, E, and F) are constructed using different matching networks. The values of the lumped elements of each filter are listed in Fig. 3c. The functions of $L_{\mathrm{s} 1}, L_{\mathrm{s} 2}, L_{\mathrm{p} 1}$, and $L_{\mathrm{p} 2}$ are similar to those in the firstorder case. Filter D has the largest $L_{\mathrm{s} 1}, L_{\mathrm{s} 2}, L_{\mathrm{p} 1}$, and $L_{\mathrm{p} 2}$, so it has the widest FBW but the smallest out-of-band rejection (Fig. 3b). Filter $\mathrm{F}$ has the smallest $L_{\mathrm{s} 1}, L_{\mathrm{s} 2}, L_{\mathrm{p} 1}$, and $L_{\mathrm{p} 2}$, which corresponds to the smallest FBW but the highest out-of-band rejection. Filter $E$ has intermediate values of $L_{\mathrm{s} 1}, L_{\mathrm{s} 2}, L_{\mathrm{p} 1}$, and $L_{\mathrm{p} 2}$, and its FBW and out-ofband rejection are between Filter Ds and Filter Fs. Overall, the second-order filters exhibit much better out-of-band rejection and roll-off than the first-order filters.

The bandwidth widening effect of the second-order wideband filters is also researched and compared in Fig. 3d. Filter D has the highest BWF of 4.6 because it uses the largest $L_{\mathrm{s} 1}, L_{\mathrm{s} 2}, L_{\mathrm{p} 1}$, and $L_{\mathrm{p} 2}$. This BWF could be further increased if higher $L_{\mathrm{s} 1}, L_{\mathrm{s} 2}, L_{\mathrm{p} 1}$, and $L_{\mathrm{p} 2}$ were applied. Of course, the compromise will be the degradation of out-ofband rejection. Filter $\mathrm{F}$ has the smallest BWF of 2.2, but its out-of-band rejection is as high as $76 \mathrm{~dB}$. Compared with the first-order wideband filter $\mathrm{C}$, which has the same BWF of 2.2, the second-order wideband filters achieve much better out-of-band rejection performance.

The MEMS resonators in the proposed first-order and second-order filters are constructed by the MBVD model. There are no specific requirements of the types of resonators. The MEMS resonators could be various MEMS resonators, such as AlN Lamb wave resonators, SAW resonators, FBARs, or $\mathrm{LiNbO}_{3} \mathrm{~A} 1$ resonators. To validate the feasibility and achieve multiple-frequency filters, the following section will replace the general

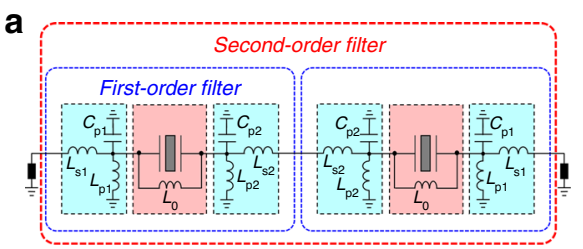

\begin{tabular}{c|c|c|c} 
C & \multicolumn{1}{|c}{} \\
\hline Parameters & Filter D & Filter E & Filter F \\
\hline$L_{0}(\mathrm{nH})$ & 750 & 750 & 750 \\
\hline$L_{\mathrm{s} 1}(\mathrm{nH})$ & 379 & 315 & 211 \\
$L_{\mathrm{p} 1}(\mathrm{nH})$ & 1650 & 423 & 355 \\
$C_{\mathrm{p} 1}(\mathrm{pF})$ & 1.6 & 2.7 & 3.6 \\
$L_{\mathrm{s} 2}(\mathrm{nH})$ & 1583 & 1242 & 783 \\
$L_{\mathrm{p} 2}(\mathrm{nH})$ & 157 & 96 & 31 \\
$C_{\mathrm{p} 2}(\mathrm{pF})$ & 3.2 & 5.1 & 15.4 \\
\hline FBW & $7.8 \%$ & $6.7 \%$ & $3.7 \%$ \\
Rej $(\mathrm{dB})$ & 55 & 65 & 76 \\
\hline d & & & \\
\hline Filter & Resonator & $\mathrm{FBW}$ & $\mathrm{BWF}$ \\
\hline D, this work & $1.7 \%$ & $7.8 \%$ & 4.6 \\
E, this work & $1.7 \%$ & $6.7 \%$ & 3.9 \\
F, this work & $1.7 \%$ & $3.7 \%$ & 2.2 \\
Ladder & $1.7 \%$ & $0.78 \%$ & 0.45 \\
Lattice & $1.7 \%$ & $0.98 \%$ & 0.56 \\
\hline
\end{tabular}

Fig. 3 The topology and performance of the proposed second-order filter. a Proposed second-order wideband filter and its simulated filtering response. $\mathbf{b}$ Simulated filtering performance of the three second-order wideband filters with different matching networks or matching lumped elements. Filter $D$ has the largest $L_{s,} L_{s 2}, L_{p}$ and $L_{p 2}$, Filter $E$ has an intermediate $L_{s 1} L_{s 2}, L_{p}$ and $L_{p 2}$, and Filter $F$ has the smallest $L_{s 1} L_{s 2}$, $L_{p}$ and $L_{p 2}$. c Comparison of the three second-order wideband filters. $\mathbf{d}$ Comparison of the bandwidth widening effect of three second-order wideband filters and conventional ladder and lattice filtering topologies 
MEMS resonator with a concrete AlN Lamb wave resonator.

\section{Material and device design AIN Lamb wave resonator}

AlN has emerged as the most suitable material for the transduction of acoustic waves because of its excellent performance and manufacturability. Engineered AlN has shown desirable properties of large phase velocity, high thermal conductivity, low acoustic loss, and relatively small temperature coefficients of frequency $(\mathrm{TCFs})^{19}$, which make it preferable for resonator applications. AlN S0 Lamb wave resonators have attained great success in terms of high resonant frequency, great power-handling capability, high $Q$, and low-frequency drift $^{30-32}$. Most importantly, Lamb wave resonators make it possible to integrate multiple-frequency resonators ${ }^{26}$ or filters $^{33}$ on piezoelectric films of the same thickness. However, the relatively low electromechanical coupling $(<2 \%)$ of the AlN S0 Lamb wave resonators limits their use in RF wideband filters. As analyzed, the proposed wideband filtering topologies can significantly extend the FBWs, so in this section, AlN MEMS wideband filters will be designed and implemented.

As illustrated in Fig. 4a, the designed AlN SO Lamb wave resonator is comprised of top interdigitated transducers (IDTs), a suspended AlN thin film, and a bottom electrode (BE). The top IDTs are alternatingly connected to the ground and RF signal, and the $\mathrm{BE}$ is electrically floating. The resonator is designed with four tethers at the four corners to increase the structure robustness and power-handling capability. The resonant frequency of the intended S0 mode is designed to be approximately $450 \mathrm{MHz}$. The BE and top IDTs are chosen to be $100 \mathrm{~nm}$ $\mathrm{Pt}$ and $150 \mathrm{~nm} \mathrm{Al}$, respectively. The middle AlN thin film is approximately $1 \mu \mathrm{m}$ thick. The pitch width of the IDTs is $10 \mu \mathrm{m}$, which forms the designed resonant frequency of approximately $450 \mathrm{MHz}$. The parameters of the AlN S0 Lamb wave resonator are described in Fig. 4c.

Figure $4 \mathrm{~b}$ shows the fabrication process of the AlN S0 resonator. It starts with a high-resistivity $\mathrm{Si}$ wafer. $\mathrm{Pt}$ $(100 \mathrm{~nm})$ is deposited by evaporation and then lifted off with negative photoresist AZ5214E. Then, $1 \mu \mathrm{m}$ thick AlN is reactively sputtered, followed by IDT lithography with photoresist SPR220. The $150 \mathrm{~nm} \mathrm{Al}$ is sputtered and lifted off as the top IDTs. A hard mask of $\mathrm{SiO}_{2}$ is chosen to define the AlN film by inductively coupled plasmareactive ion etching (ICP-RIE). After the ICP-RIE, the remaining $\mathrm{SiO}_{2}$ is removed in $\mathrm{HF}$.

Finally, the resonator is exposed to $\mathrm{XeF}_{2}$ for release. Figure $5 \mathrm{a}-\mathrm{d}$ shows the scanning electron microscope (SEM) images of the fabricated device and its zoomed-in structures.

The fabricated resonator was measured in dry air with an Agilent PNA-L 5230A at room temperature. The measured admittance result is shown in Fig. 5e. The resonator has a measured resonant frequency at $446 \mathrm{MHz}$, an electromechanical coupling of $0.94 \%$ and a $Q$ of 1500 .

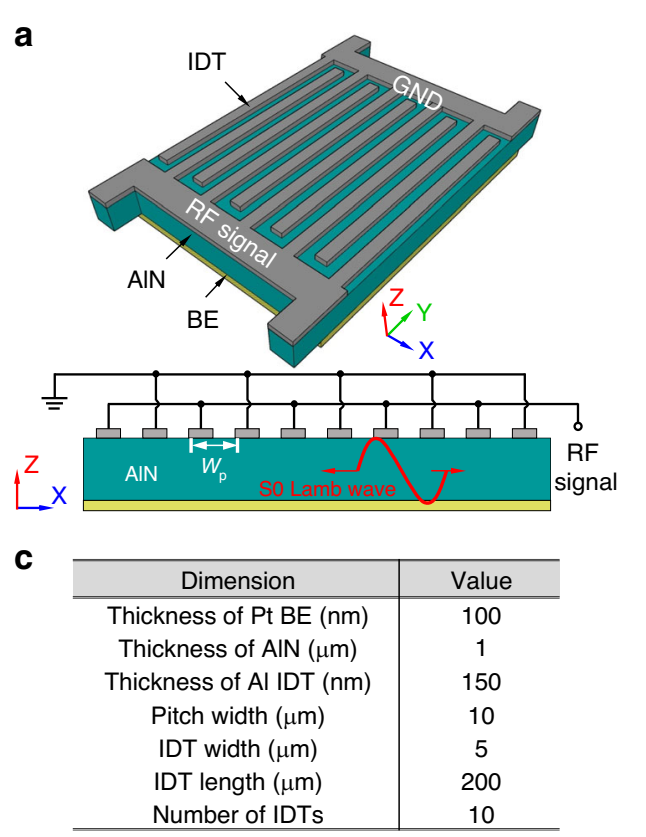

b

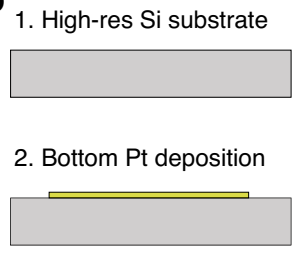

3. AIN deposition

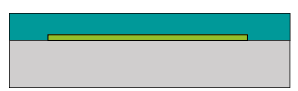

4. IDT lithography

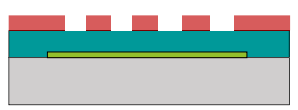

5. Deposition of IDTs

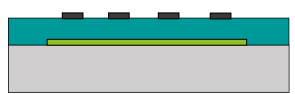

6. Definition of $\mathrm{SiO}_{2}$ mask

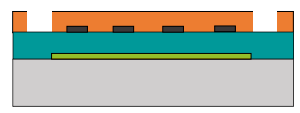

7. ICP-RIE of AIN

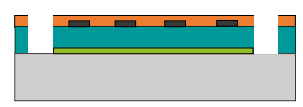

8. $\mathrm{SiO}_{2}$ removal

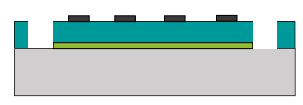

9. Device release by $\mathrm{XeF}_{2}$
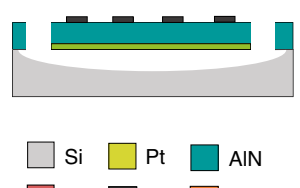

Fig. 4 Fabrication process of the AIN Lamb wave resonator. a Prospective view and cross-sectional view of the AIN Lamb wave resonator. The resonator consists of three layers: bottom electrode, AIN thin film, and top IDTs. The Lamb wave is excited in the stack. $\mathbf{b}$ Fabrication process of the AIN SO Lamb wave resonator. c Dimensions of the designed AIN SO Lamb wave resonator 

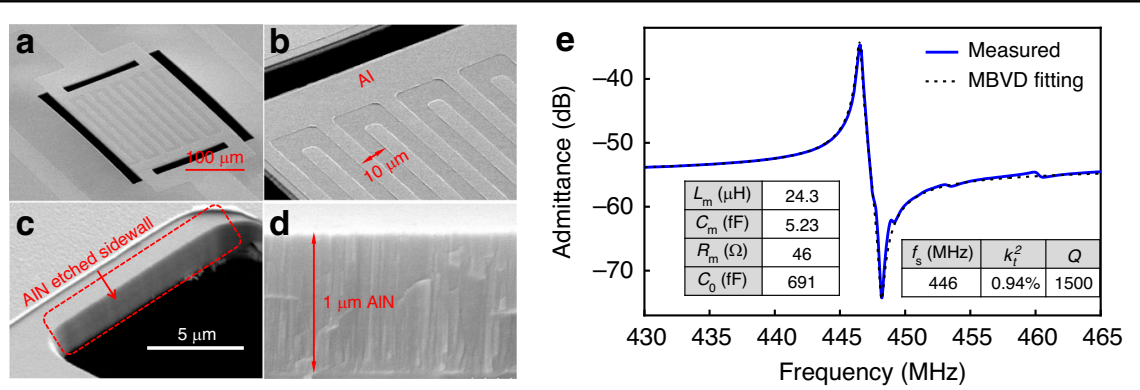

Fig. 5 SEM image and measured response of fabricated AIN SO Lamb wave resonator. SEM images of the (a) fabricated AIN SO Lamb wave resonator, (b) Al IDTs, (c) etched AIN sidewall, and (d) $1 \mu \mathrm{m}$ AIN crystal. e Measured admittance response of the fabricated AIN S0 Lamb wave resonator. The measured response is fitted by the MBVD model

The resonator's measured response is then fitted by the MBVD model. The fitted MBVD $L_{\mathrm{m}}, C_{\mathrm{m}}, R_{\mathrm{m}} C_{0}$ parameters are listed in the inset of Fig. 5e and will be used in the AlN S0 first- and second-order hybrid wideband filters.

\section{AIN SO first-order wideband filter}

The principle of the first-order wideband filter has been introduced in the Method and Modeling section. In this section, we will replace the general MEMS resonator in Fig. 1d with the fabricated AlN S0 Lamb wave resonator, as indicated in Fig. 6a. The AIN SO Lamb wave resonator is modeled by using the MBVD circuit lumped elements listed in the inset of Fig. 5e. According to Eq. (2), the parallel $L_{0}$ is calculated to be $188 \mathrm{nH}$. For the other three-lumped elements, $L_{\mathrm{s}}$ and $L_{\mathrm{p}}$ are chosen to be $160 \mathrm{nH}$ and $145 \mathrm{nH}$, respectively, and $C_{\mathrm{p}}$ is computed to be $1.7 \mathrm{pF}$ (shown in the inset of Fig. 6b). Generally, inductors and capacitors are lossy due to their finite Qs. According to the datasheets of current lumped-element vendors, the $Q \mathrm{~s}$ of $L_{0}, L_{\mathrm{s}}, L_{\mathrm{p}}$, and $C_{\mathrm{p}}$ are assumed to be $87,87,92$, and 400 , respectively.

The simulation result of the AlN S0 first-order wideband filter is illustrated in Fig. 6b. The filter is centered at $446 \mathrm{MHz}$ with a low IL of $1.8 \mathrm{~dB}$, a high FBW of $5.6 \%$, and an out-of-band rejection of $24 \mathrm{~dB}$. As noted, the electromechanical coupling of the AlN S0 resonator is only $0.94 \%$. However, it reveals a very high BWF of 6 after implementing the first-order filter topology.

The out-of-band rejection of the AlN S0 first-order wideband filter is moderate. For improvement, an AlN S0 second-order wideband filter is then evaluated below.

\section{AIN S0 second-order wideband filter}

The topology of the AlN S0 second-order filter is similar to that of the second-order filter in Fig. 3a. Shown in Fig. 6c, the AlN S0 second-order wideband filter consists of two series of AlN S0 first-order filters. $L_{0}$ is the same as that in the AlN S0 first-order filter. The lumped elements $\left(L_{\mathrm{s} 1}, L_{\mathrm{p} 1}, C_{\mathrm{p} 1}, L_{\mathrm{s} 2}, L_{\mathrm{p} 2}\right.$, and $\left.C_{\mathrm{p} 2}\right)$ are adjusted accordingly for better impedance matching. Figure $6 \mathrm{~d}$ shows the simulated performance of the AlN S0 second-order filter. The values of the lumped elements used in the filter are provided in the inset of Fig. $6 \mathrm{~d}$. The second-order filter is also centered at $446 \mathrm{MHz}$. It has an IL of $2.6 \mathrm{~dB}$, a wide FBW of 5.1\%, and very high out-of-band rejection (e.g., $43 \mathrm{~dB}$ at $300 \mathrm{MHz}$ ). The $2.6 \mathrm{~dB}$ IL is higher than the $1.8 \mathrm{~dB}$ in the AIN S0 first-order filter because of the additional cascaded loss from the lumped elements. This $2.6 \mathrm{~dB} \mathrm{IL}$ can be reduced if high- $Q$ lumped elements are available or at higher frequencies where high- $Q$ lumped elements are attainable. In short, the second-order filters have much higher out-of-band rejection than the first-order filters, and the payoff is the complexity of the circuit as well as the cost of extra lumped components.

\section{Results and discussion}

\section{Implementation of the AIN MEMS filter}

Due to the resource limitation and the similarity among the proposed filters, we choose only the AlN S0 first-order wideband filter to implement. The dimensions of the fabricated AlN S0 first-order wideband filter are listed in Fig. 4c. The filter is implemented by first fabricating the AlN S0 Lamb wave resonator and then integrating it with the matching networks on a printed circuit board (PCB). The fabrication of the AlN S0 resonator utilizes a process described in detail in Fig. 4b. The AlN S0 resonators are fabricated on a resonator chip. It is then cut into smaller pieces for later PCB mounting.

As shown in Fig. 7a, the filter assembly process starts with PCB preparation, which includes size planning and surface cleaning. The chosen PCB is based on an FR4 with a thickness of $1.524 \mathrm{~mm}$, a dielectric constant $\left(\varepsilon_{r}\right)$ of 4.8 , a dielectric loss tangent $\tan \left(\delta_{\mathrm{D}}\right)$ of 0.008 , and a copper cladding thickness of $18 \mu \mathrm{m}$. Integration generally requires wire bonding (step 7) at $120^{\circ} \mathrm{C}$. However, the copper cladding would be oxidized very quickly and make the bonding fail. To avoid this, $50 \mathrm{~nm}$ platinum $(\mathrm{Pt})$ and $250 \mathrm{~nm}$ gold $(\mathrm{Au})$ are continuously evaporated on the 


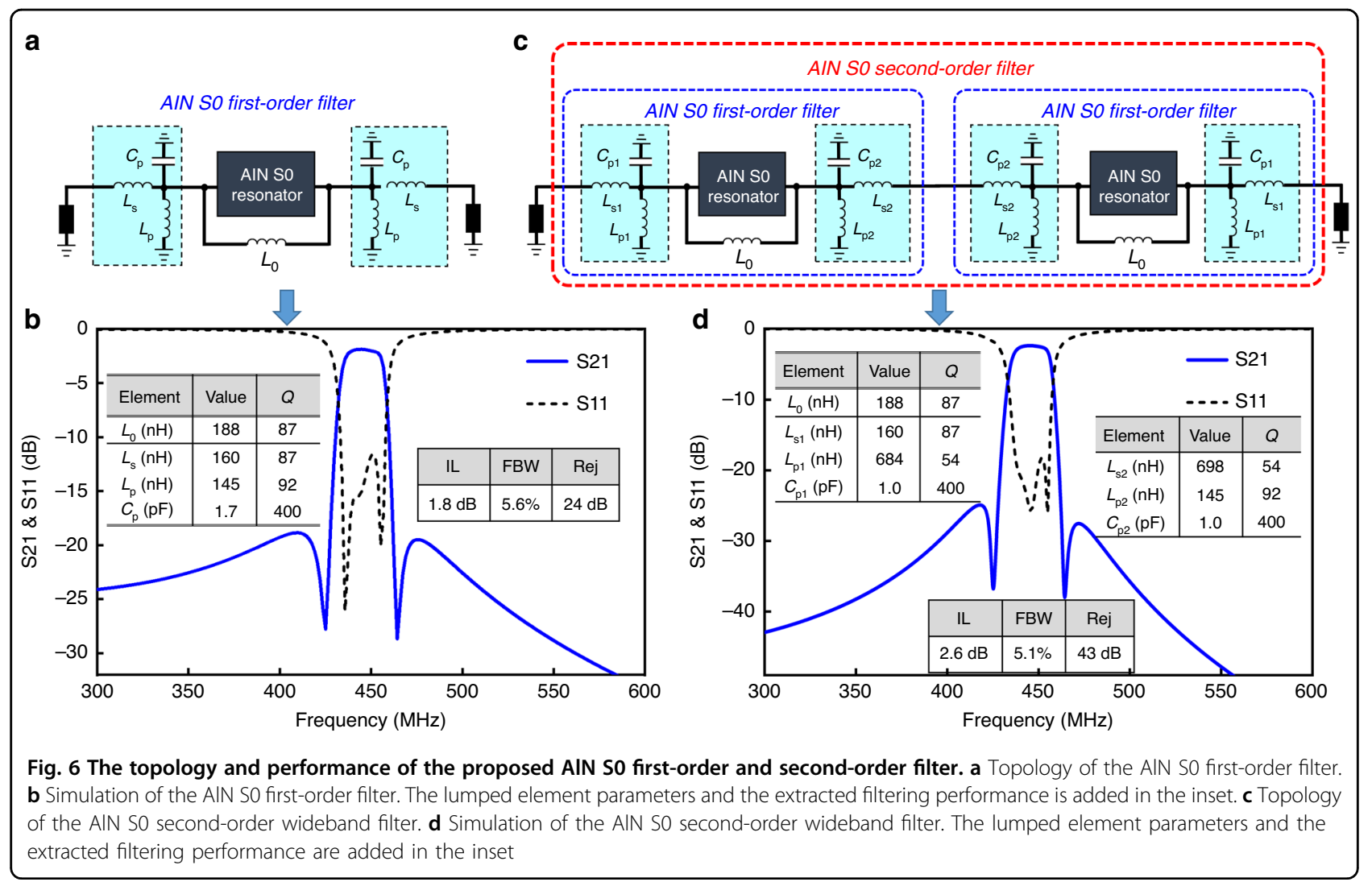

PCB. The Pt works as an adhesion layer, while the $\mathrm{Au}$ layer has the two-fold purpose of antioxidation and easy bonding (a gold substrate usually provides the best bonding ease). Subsequently, the copper is milled to form the signal traces and bonding pads. For easy bonding, a bonding box with a depth of $600 \mathrm{~nm}$ is opened in the center of the PCB. The bounding box helps the top surfaces of the resonator chip and the PCB stay on the same plane. It also ensures that the bonding wires do not touch the corner of the chip. The lumped elements of the surface mount inductors and capacitors are then soldered on the PCB with a hot air gun and soldering paste. The resonator chip is attached to the bonding box and then wire bonded to the signal traces on the PCB. Finally, two SMA connectors are installed for measurement.

The PCB assembly has considerable influence on the filter. There are several aspects we need to monitor so that minimal PCB influence is introduced. First, the parasitic effect from the PCB should be reduced. In our circuit model in Fig. 6a, the filter contains the lumped elements and AIN MEMS resonators only. There is no parasitic content in the filter circuit. However, in the real case, when we assemble the filter to the $\mathrm{PCB}$, the parasitic effect always exists and influences the filter performance. For low-frequency filters, the parasitic effect is not that significant and can be compensated by tuning the three- lumped elements (tuning $C_{\mathrm{p}}$ would be a good option). For high-frequency filters, the parasitic effect becomes dominant, which requires an electromagnetic environment to be applied to the filter circuit. Second, the grounding pads on the PCB are key. Grounding is critical because it generates different levels of parasitic capacitance. To achieve a good PCB assembly, the grounding pads on the PCB must be carefully designed so that minimal parasitic capacitance is generated. Third, the bonding wires introduce parasitics. Bonding wires can be thought of as a kind of inductor, which can also exert an influence on the filter. One has to plan the assembly beforehand so that the least length of bonding wire is needed.

Figure 7 demonstrates the assembly process and images of the fabricated filter with some integration details. The fabricated filter (Fig. 7b) has dimensions of $1.85 \mathrm{~cm} \times$ $0.85 \mathrm{~cm}$. This dimension is smaller than that of most microstrip filters but still larger than those of pure acoustic filters (SAW or BAW filters). The main causes of the relatively large size are die-level bonding and manual mounting, which require additional operation space. By using a more compact flip-chip approach that involves chip-scale lumped elements, the form factor of the filter can be substantially improved. Figure $7 \mathrm{c}$ shows the AlN resonator chip featuring arrays of resonators. The target AlN S0 resonator is at the top center as circled. Figure $7 d$ 


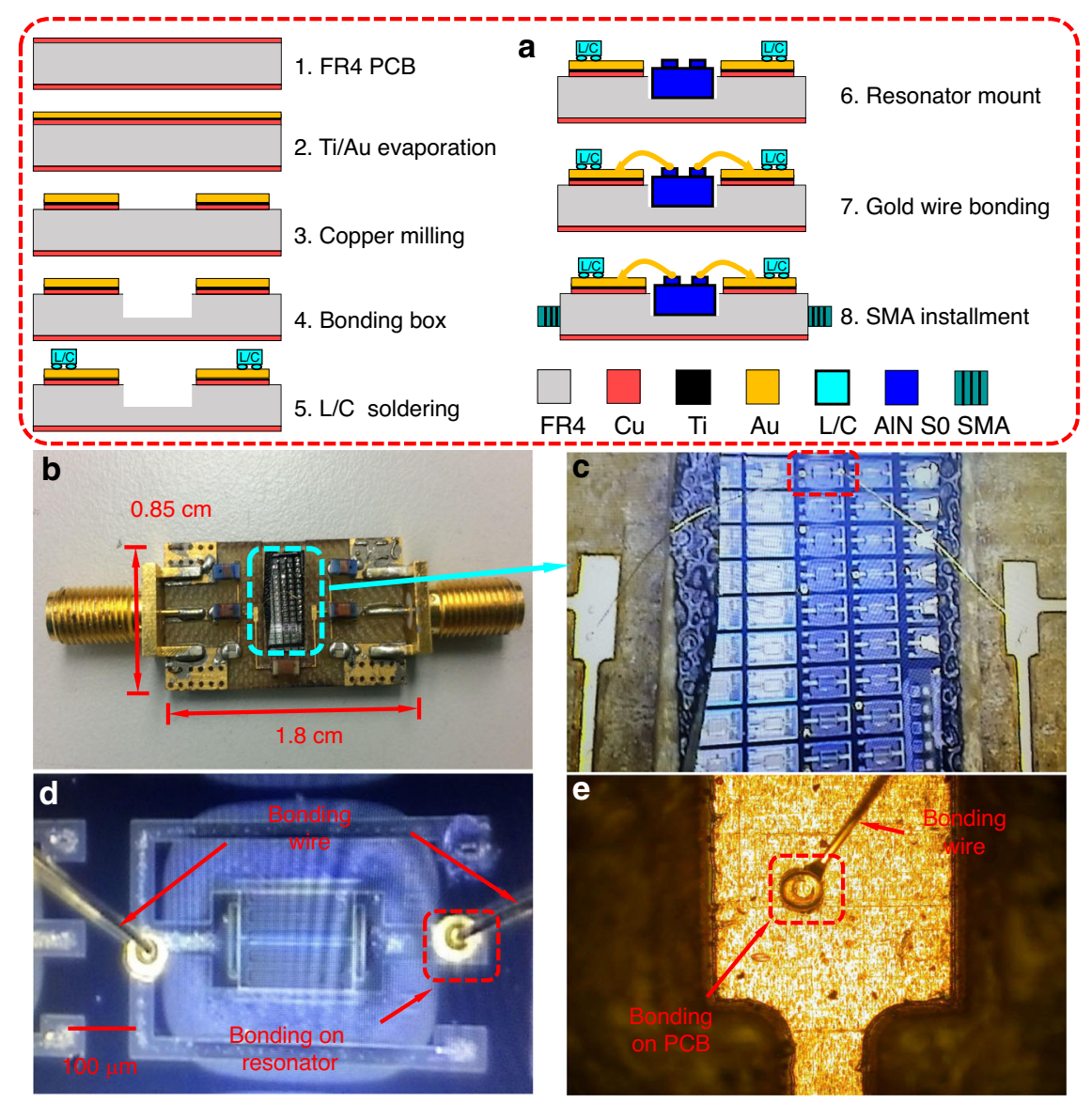

Fig. 7 Assembly process of the proposed AIN S0 first-order filter. a Assembly process of the AIN SO first-order wideband filter. $\mathbf{b}$ Fabricated AIN SO first-order wideband filter. c Mounted AIN resonator chip. $\mathbf{d}$ Magnified view of the bonded AIN. e Bonded wire on the PCB
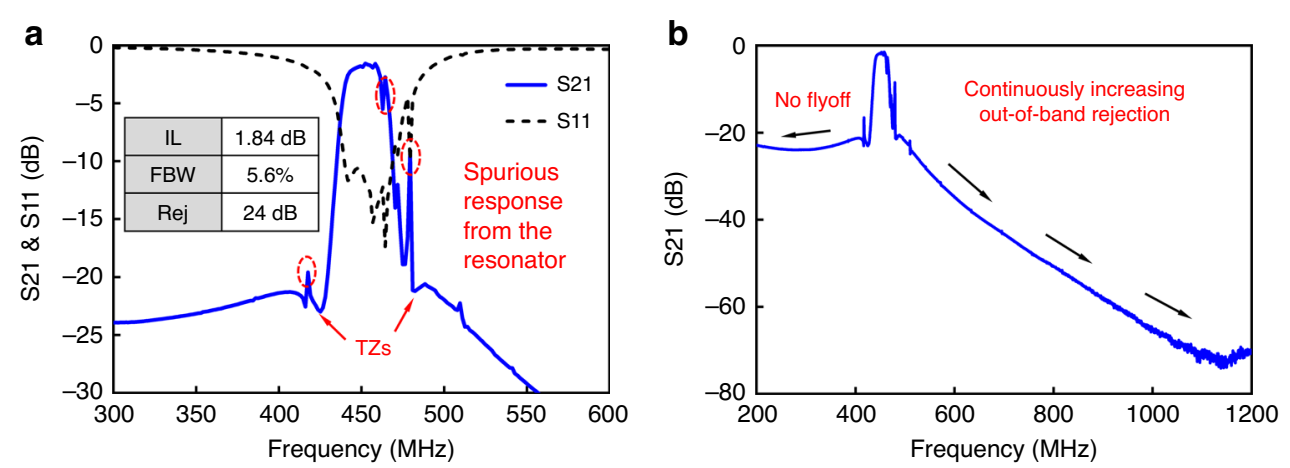

Fig. 8 Measured response of the fabricated AIN S0 first-order filter. a Measured response (S21 and S11) of the fabricated AIN S0 first-order filter from $300 \mathrm{MHz}$ to $600 \mathrm{MHz}$. b Measured response (S21) of the same filter from 200 to $1200 \mathrm{MHz}$

is the magnified view of the bonded AlN S0 resonator. The two bonding wires connect the two ports (input and output ports) of the resonator. The two wires start from the resonator and end on the bonding pads on the PCB, as shown in Fig. 7e.

\section{Characterization results}

The fabricated AlN S0 first-order wideband filter is measured by an Agilent PNA-L 5230 A analyzer in dry air and at room temperature. The measurement results are shown in Fig. 8a. The filter demonstrates a low IL of 
$1.84 \mathrm{~dB}$, a wide FBW of $5.6 \%$, and out-of-band rejection of $24 \mathrm{~dB}$. Overall, the measurement agrees well with the simulation results shown in Fig. 6b. As noted, there are two mismatches: the depth of the two TZs and the spurious response. The measurement has shallower TZs than the simulation. This can be attributed to the fact that the actual $Q$ of $L_{0}$ is lower than the simulated $Q$ provided by the vendors. Generally, a higher $Q_{\mathrm{L} 0}$ gives a deeper TZ depth. The spurious response in the measurement comes from the spurious modes of the resonator. The simulation adopts the MBVD model, which does not incorporate spurious modes. This explains why we see the spurious response in the measurement only. Techniques have been reported to suppress the spurious modes in AlN Lamb wave resonators ${ }^{34-37}$. By applying these spurious mode suppression techniques, the spurious response of the filter could be eradicated.

Figure $8 \mathrm{~b}$ plots the measured response of the filter over a wider frequency range between 200 and $1200 \mathrm{MHz}$. As seen, the out-of-band rejection on the left has no fly off. This advantage overcomes the long-existing fly off issues in microstrip filters or purely acoustic filters. In addition, the out-of-band rejection on the right continuously increases up to $75 \mathrm{~dB}$. Additionally, there are no spurious harmonic responses. This merit overtakes its counterparts of microstrip filters and purely acoustic filters.

Finally, it is worthwhile to notice the bandwidth widening capability of the achieved filter. The coupling of the AlN S0 Lamb wave resonator is $0.94 \%$, and the achieved FBW of the filter is $5.6 \%$. Therefore, the BWF is calculated to be 6.0 , which is approximately 12 times higher than that of the conventional ladder or lattice topologies. As predicted from Eq. (3), a higher FBW can be achieved by implementing this bandwidth widening technique on resonators with a higher piezoelectric coupling factor.

\section{Conclusion}

Wideband MEMS filters have been analyzed, designed, fabricated and characterized in this work. Two filtering topologies (first- and second-order) are first proposed and researched. Then, AlN S0 Lamb wave resonators are applied to the filtering topologies and show excellent performance of low IL, wide bandwidth, and high out-ofband rejection. To validate the simulated filters, the AlN S0 first-order filter is chosen for implementation, which progresses from the resonator fabrication to the final filter assembly. The demonstrated AlN S0 first-order filter has a high FBW of $5.6 \%$, a low IL of $1.84 \mathrm{~dB}$, and an out-of-band rejection larger than $24 \mathrm{~dB}$. Considering the low coupling $(0.94 \%)$ of the AlN S0 resonator, the BWF is as high as 6, which is approximately 12 times higher than that of the ladder or lattice topologies. Furthermore, the demonstrated AlN So first-order filter features far-frequency suppression without spurious responses. The presented hybrid filters hold great potential for various filtering applications, especially for the current $5 \mathrm{G}$ NR bands of n77, n78, and n79.

Moreover, very recently, high-frequency on-chip rolledup inductors with miniaturized sizes have been reported $^{38,39}$. The self-rolled-up inductor can achieve a maximum $Q$ factor of over 12 at $3.5 \mathrm{GHz}$ and $10 \mathrm{nH}$ inductance with a footprint area of only $15 \times 19 \mu \mathrm{m}^{2}$, which is $0.1 \%$ of that of planar spiral inductors ${ }^{38}$. Their fabrication process could be incorporated with that of the Lamb wave resonators. In addition, it has been shown that piezoelectric resonators can be integrated with capacitors on the same membrane ${ }^{40}$, especially on $\mathrm{AlN}^{41}$, since the AlN thin film sputtering and resonator device fabrication process is compatible with CMOS ICs. Therefore, it is possible to achieve the inductances $L_{0}, L_{\mathrm{s}}$, and $L_{\mathrm{p}}$ with these rolled-up inductors and the capacitance $C_{\mathrm{p}}$ with the AlN Lamb wave resonators and finally attain multiplefrequency on-chip hybrid filters. This will be our future development.

\section{Acknowledgements \\ This work was supported by the Science and Technology Commission of Shanghai Municipality (Shanghai Pujiang Program 18PJ1408300), Natural Science Foundation of Shanghai (19ZR1477000) and National Science Foundation of China (61874073).}

\section{Author details}

${ }^{1}$ Department of Electrical and Computer Engineering, University of Illinois at Urbana-Champaign, Urbana, IL 61820, USA. ${ }^{2}$ School of Information Science and Technology (SIST), ShanghaiTech University, Shanghai 201210, China. ${ }^{3}$ University of Chinese Academy of Sciences, No. 19(A) Yuquan Road, Shijingshan District, Beijing, China

\section{Author contributions}

A.G. performed experimental design, simulation and analysis; K.L. performed simulation, analysis, and writing; J.L. and T.W. discussed the manuscript and analysis.

Conflict of interest

The authors declare that they have no conflict of interest.

Received: 29 December 2019 Revised: 5 May 2020 Accepted: 6 May 2020 Published online: 07 September 2020

\footnotetext{
References

1. Plessky, V. et al. $5 \mathrm{GHz}$ laterally-excited bulk-wave resonators (XBARs) based on thin platelets of lithium niobate. Electron. Lett. 55, 98-100 (2019).

2. Gao, A., Zou, J. \& Gong, S. A 3.5 GHz AlN S1 Lamb mode resonator. In 2017 IEEE International Ultrasonics Symposium 1-4 (2017).

3. Cassella, C., Hui, Y., Qian, Z., Hummel, G. \& Rinaldi, M. Aluminum nitride crosssectional Lamé mode resonators. IEEE J. Microelectromech. Syst. 25, 275-285 (2016).

4. Cassella, C. et al. Super high frequency aluminum nitride two-dimensionalmode resonators with kt2 exceeding 4.9\%. IEEE Microw. Wirel. Compon. Lett. 27, 105-107 (2017).

5. Colombo, L. et al. Investigation of $20 \%$ scandium-doped aluminum nitride films for MEMS laterally vibrating resonators. In 2017 IEEE International Ultrasonics Symposium 1-4 (2017).
} 
6. Zhu, Y. et al. ScAlN-based LCAT mode resonators above $2 \mathrm{GHz}$ with high FOM and reduced fabrication complexity. IEEE Electron Device Lett. 38, 1481-1483 (2017).

7. Wang, N. et al. Over $10 \%$ of keff2 demonstrated by $2-\mathrm{GHz}$ spurious mode-free $\mathrm{Sc}_{0.12} \mathrm{Al}_{0.88} \mathrm{~N}$ laterally coupled alternating thickness mode resonators. IEEE Electron Device Lett. 40, 957-960 (2019).

8. Psychogiou, D., Gómez-garcía, R., Sánchez, R. \& Peroulis, D. Hybrid acousticwave-lumped-element resonators (AWLRs) for high-bandpass filters with quasi-elliptic frequency response. IEEE Trans. Microw. Theory Tech. 63, 2233-2244 (2015).

9. Psychogiou, D., Gómez-garcía, R., Sánchez, R. \& Peroulis, D. Coupling-matrixbased design of high-bandpass filters using acoustic-wave lumped-element resonator (AWLR) modules. IEEE Trans. Microw. Theory Tech. 63, 4319-4328 (2015).

10. Lu, X., Mouthaan, K. \& Soon, Y. Wideband bandpass filters with SAW-filter-like selectivity using chip SAW resonators. IEEE Trans. Microw. Theory Techn. 62, 28-36 (2014).

11. Kadota, M., Ogami, T. \& Kimura, T. Wide band resonators using SH Mode of plate wave on $\mathrm{LiNbO}_{3}$. In 2012 IEEE International Ultrasonics Symposium. 563-567 (2012).

12. Yang, Y., Gao, A., Lu, R. \& Gong, S. 5 GHz lithium niobate MEMS resonators with high FoM of 153. In 2017 IEEE International Ultrasonics Symposium. $942-945$ (2017).

13. Song, Y. \& Gong, S. Spurious mode suppression in SHO lithium niobate laterally vibrating MEMS resonators. In 2015 IEEE International Electron Devices Meeting. 18-5 (2017).

14. Song, Y. \& Gong, S. Wideband spurious-free lithium niobate RF-MEMS filters. J. Microelectromech. Syst. 26, 820-828 (2017).

15. Kadota, M. \& Tanaka, S. Solidly mounted ladder filter using shear horizontal wave in $\mathrm{LiNbO}_{3}$. In 2016 IEEE International Ultrasonics Symposium. 1-4 (2016).

16. Mateen, F., Boales, J., Erramilli, S. \& Mohanty, P. Micromechanical resonator with dielectric nonlinearity. Microsyst. Nanoeng. 4, 1-7 (2018).

17. Mateen, F., Maedler, C., Erramilli, S. \& Mohanty, P. Wireless actuation of micromechanical resonators. Microsyst. Nanoeng. 2, 1-6 (2016).

18. Boales, J. A., Mateen, F. \& Mohanty, P. Optical wireless information transfer with nonlinear micromechanical resonators. Microsyst. Nanoeng. 3, 1-6 (2017).

19. Qian, Z. et al. Graphene-aluminum nitride NEMS resonant infrared detector. Microsyst. Nanoeng. 2, 1-7 (2016).

20. Fischer, A. C. et al. Integrating MEMS and ICs. Microsyst. Nanoeng. 1, 1-16 (2015).

21. Sano, K., Karasawa, R. \& Yanagitani, T. High electromechanical coefficient kt2 $=19 \%$ thick ScAlN piezoelectric films for ultrasonic transducer in low frequency of $80 \mathrm{MHz}$. In 2017 IEEE International Ultrasonics Symposium. 1-4 (2017).

22. Carlotti, G., Sadhu, J. \& Dumont, F. Dependence of the different elastic constants of SCAIN films on Sc content: a Brillouin scattering study with polarization analysis. In 2017 IEEE International Ultrasonics Symposium. 1-5 (2017).

23. Matloub, R. et al. Piezoelectric Al1-XSc $\mathrm{N}$ thin films: a semiconductor compatible solution for mechanical energy harvesting and sensors Appl. Phys. Lett. 102, 152903 (2013).

24. Piazza, G., Stephanou, P. \& Pisano, A. Piezoelectric aluminum nitride vibrating contour-mode MEMS resonators. J. Microelectromech. Syst. 15, 1406-1418 (2006).
25. Lin, C. M., Yantchev, V., Zou, J., Chen, Y. \& Pisano, A. Micromachined one-port aluminum nitride Lamb wave resonators utilizing the lowest order symmetric mode. J. Microelectromech. Syst. 23, 78-91 (2014).

26. Zou, J., Lin, C.-M., Gao, A. \& Pisano, A. P. The multi-mode resonance in AIN Lamb wave resonators. IEEE J. Microelectromech. Syst. 27, 973-984 (2018).

27. Yang, Q., Pang, W., Zhang, D. \& Zhang, H. A modified lattice configuration design for compact wideband bulk acoustic wave filter applications. Micromachines 7, 133 (2016).

28. Gong, S. \& Piazza, G. Design and analysis of lithium-niobate-based high electromechanical coupling RF-MEMS resonators for wideband filtering. IEEE Trans. Microw. Theory Tech. 61, 403-414 (2013).

29. Zheng, X. Q., Lee, J. \& Feng, P. X. L. Hexagonal boron nitride nanomechanical resonators with spatially visualized motion. Microsyst. nanoengineering $\mathbf{3}, 17038$ (2017).

30. Gao, A. \& Gong, S. Harnessing mode conversion in AIN laterally vibrating resonators for spurious mode suppression. IEEE J. Microelectromechanical Syst. 25, 450-458 (2016).

31. Yantchev, V. \& Katardjiev, I. Design and fabrication of thin film Lamb wave resonators utilizing longitudinal wave and interdigital transducers. In 2005 IEEE International Ultrasonics Symposium. 1580-1583 (2005).

32. Gao, A., Zou, J. \& Wu, T. Narrowband impedance transformer with extremely high transformation ratio of 200. IEEE Electron Device Lett. 40, 1820-1823 (2019).

33. Piazza, G., Stephanou, P. J. \& Pisano, A. P. Single-chip multiple-frequency ALN MEMS filters based on contour-mode piezoelectric resonators. IEEE J. Microelectromech. Syst. 16, 319-328 (2007).

34. Li, W.-C., Lin, Y., Kim, B., Ren, Z. \& Nguyen, C.-T. Quality factor enhancement in micromechanical resonators at cryogenic temperatures. In 2009 IEEE International Solid-State Sensors, Actuators and Microsystems Conference. 1445-1448 (2009).

35. Olsson, R. H., Nguyen, J., Pluym, T. \& Hietala, V. M. A method for attenuating the spurious responses of aluminum nitride micromechanical filters. J. Microelectromech. Syst. 23, 1198-1207 (2014).

36. Giovannini, M., Yazici, S., Kuo, N. K. \& Piazza, G. Apodization technique for spurious mode suppression in AlN contour-mode resonators. Sens. Actuators A: Phys. 206, 42-50 (2014).

37. Zhang, $\mathrm{H}$. et al. Transverse mode spurious resonance suppression in Lamb wave MEMS resonators: theory, modeling, and experiment. IEEE Trans. Electron Devices 62, 3034-3041 (2015).

38. Huang, W. et al. On-chip inductors with self-rolled-up $\mathrm{SiN}_{x}$ nanomembrane tubes: a novel design platform for extreme miniaturization. Nano Lett. 12, 6283-6288 (2012).

39. $\mathrm{Yu}, \mathrm{X}$. et al. Ultra-small, high-frequency, and substrate-immune microtube inductors transformed from 2D to 3D. Sci. Rep. 5, 9661-9667 (2015).

40. He, Y., Bahr, B., Si, M., Ye, P. \& Weinstein, D. A tunable ferroelectric based unreleased RF resonator. Microsyst. Nanoeng. 6, 1-7 (2020).

41. Liang, J., Zhang, H., Zhang, D., Zhang, H. \& Pang, W. Lamb wave AIN micromechanical filters integrated with on-chip capacitors for RF front-end architectures. IEEE J. Electron Devices Soc. 3, 361-364 (2015). 